\title{
A syndromic extreme insulin resistance caused by biallelic POC1A mutations in exon 10
}

\author{
Elisa Giorgio",*, Elisa Rubino 2,3, *, Alessandro Bruselles4, Simone Pizzí5, Innocenzo Rainero², Sergio Duca ${ }^{3}$, \\ Fabio Sirchia ${ }^{1,6}$, Barbara Pasini, ${ }^{1,6}$, Marco Tartaglia5 and Alfredo Brusco ${ }^{1,6}$
}

${ }^{1}$ Department of Medical Sciences, University of Torino, Torino, Italy, ${ }^{2}$ Department of Neuroscience 'Rita Levi Montalcini', University of Torino, Torino, Italy, ${ }^{3}$ Koelliker Hospital, Torino, Italy, ${ }^{4}$ Department of Oncology and Molecular Medicine, Istituto Superiore di Sanità, Rome, Italy, ${ }^{5} \mathrm{Genetics}$ and Rare Diseases Research Division, Ospedale Pediatrico Bambino Gesù IRCSS, Rome, Italy, and ${ }^{6}$ Medical Genetics Unit, Città della Salute e della Scienza University Hospital, Torino, Italy

*(E Giorgio and E Rubino contributed equally to the research reported here)

Correspondence should be addressed to A Brusco

Email

alfredo.brusco@unito.it

\begin{abstract}
POC1A encodes a protein with a role in centriole assembly and stability, and in ciliogenesis. Biallelic loss-offunction mutations affecting POC1A cause SOFT syndrome, an ultra-rare condition characterized by short stature, onychodysplasia, facial dysmorphism and hypotrichosis. Using exome sequencing, we identified a homozygous frameshift mutation (c.1047_1048dupC; p.G337Rfs*25) in a patient presenting with short stature, facial hirsutism, alopecia, dyslipidemia and extreme insulin resistance. The truncating variant affected exon 10, which is retained in only two of the three POC1A-mature RNAs, due to alternative processing of the transcript. Clinical discrepancies with SOFT syndrome support the hypothesis that POC1A mutations affecting exon 10 are associated with a distinct condition, corroborating a previous hypothesis based on a similar case. Furthermore, this report provides an additional example of a genetic condition presenting with clinical heterogeneity due to alternative transcript processing. In conclusion, POC1A mutations in exon 10 should be taken into account in patients with extreme insulin resistance and short stature.
\end{abstract}

\section{Introduction}

Proteome Of Centriole 1A (POC1A) plays a central role in early phases of centriole duplication, as well as in the later steps of centriole length control, ensuring centriole integrity and proper mitotic spindle formation (1). Together with POC1B, it is an essential protein for ciliogenesis. Homozygous or compound heterozygous mutations in POC1A (\#MIM 614783) cause short Stature, Onychodysplasia, Facial dysmorphism and hypoTrichosis (SOFT) syndrome (\#MIM 614813). At present, nine families with SOFT syndrome carrying a total of seven different biallelic POC1A mutations have been described $(2,3,4$, $5,6)$. Major clinical features of SOFT syndrome include severe growth retardation, which occurs prenatally, facial
() 2017 European Society of Endocrinology Printed in Great Britain dysmorphism (dolichocephaly, triangular elongated face, prominent forehead, ear abnormalities, prominent nose and pointy chin), brachydactyly and other skeletal features (osteopenia, short carpals, metacarpals, tarsals and metatarsals tubular bones), hypoplastic finger nails and postpuberal hypotrichosis. Clinical findings in a subset of SOFT patients also include relative macrocephaly, type 2 diabetes, developmental delay and hypogonadism in males.

Patients' cells show an abnormal mitotic mechanism with multipolar spindles and clearly impaired ciliogenesis (6).

Recently, a homozygous frameshift mutation in exon 10 of the POC1A gene has been reported in a woman of Italian origin with clinical features of primordial 
dwarfism, skeletal dysplasia, facial dysmorphism, extreme dyslipidemia with insulin resistance and fatty liver, which only in part were reminiscent of SOFT syndrome (7). The authors suggested that, differently from what observed for the mutations causing SOFT syndrome, the mutation in exon 10 affects only two of the three POC1A isoforms generated by alternative processing of the POC1A transcript, preserving the isoform lacking exon 10.

Here, we report on the second patient with a homozygous truncating POC1A mutation in exon 10, corroborating the view that POC1A mutations restricted to exon 10 are not associated with SOFT syndrome but cause a distinct condition characterized by extreme insulin resistance and short stature.

\section{Subject and methods}

\section{Case description}

The proband was a 42-year-old woman of Southern Italian origin, born from first cousins. The father was healthy, while the mother presented osteoporosis and presenile Parkinson's disease with onset of the disease at 56 years of age.

Patient's first sign of disease was the appearance of acanthosis nigricans in the neck region at 7-8 years of age, initially noted by proband's mother. She had a premature menarche at age 9 years, followed by a worsening of acanthosis nigricans spreading to flexural areas including nasolabial folds, perioral region, armpits, perineum and ankles (Fig. 1A and B). She has short stature (138 cm, -4 s.D.) and normal intelligence. From childhood, she presented hands with dorsal swelling of the soft tissues (Fig. 1C). Until adolescence, her feet also showed a dorsal bulge, which dramatically reduced from 16 to 18 years of age. She also showed facial dysmorphism with micrognathia, prominent nose, low set ears, facial hirsutism that had been treated with laser therapy, alopecia and thinning hair (Fig. 1D) and eyebrows, in absence of nail and bone hypoplasia.

On clinical examination, she presented lumbar lordosis not associated either to skeletal dysplasia or 'dog-like' vertebral bodies at radiological investigations (Fig. 1E).
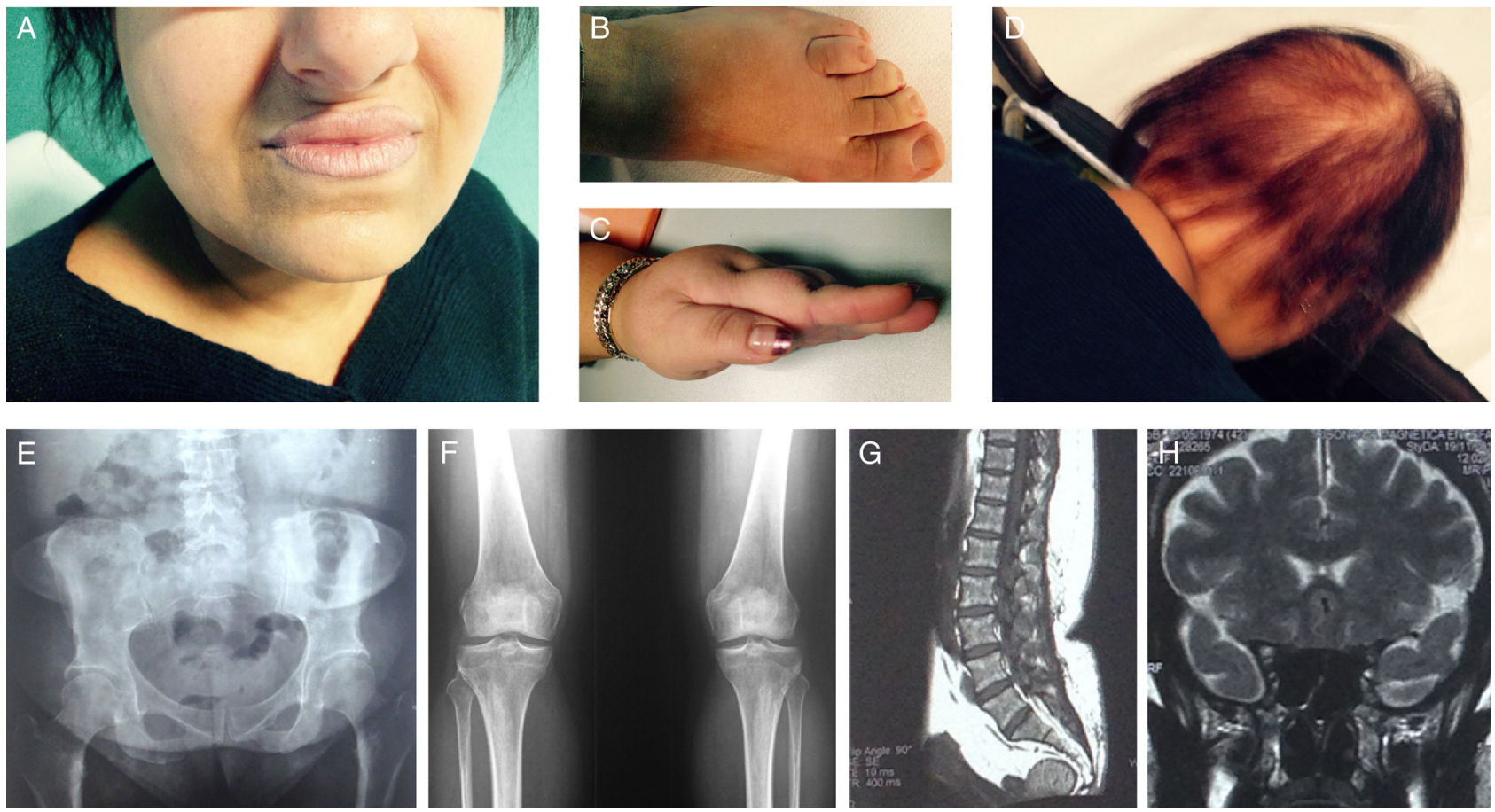

\section{Figure 1}

Clinical and neuroradiological findings. (A) Acanthosis nigricans in nasolabial folds and perioral region. (B) Acanthosis nigricans of the flexural area of the ankle; left foot with shortening of the fourth metatarsal bone. (C) Dorsal hand swelling of the soft tissues. (D) Alopecia and thinning hair. (E, F and G) X-ray images showing the hypoplastic pelvis (E). In panel F, mild bilateral knee osteoarthritis. Lumbosacral MRI (G) excluded any transitional vertebrae described in the other patient with POC1A pathogenic variant in exon 10 (7). (H) Cranial MRI did not reveal any pituitary lesion. A full colour version of this figure is available at http:// dx.doi.org/10.1530/EJE-17-0431. 
Contrariwise, a dysplastic pelvis was observed (Fig. 1E). At 41 years of age, a further radiological examination documented a mild knee arthrosis and bilateral patella osteosclerosis (Fig. 1F). Lastly, lumbosacral MRI did not show any transitional vertebrae (Fig. 1G).

At 14 years old, she was diagnosed with diabetes. She had severe fasting hyperinsulinemia, with high plasma levels of insulin after oral glucose tolerance test (120-min insulin level: $570 \mu \mathrm{U} / \mu \mathrm{L}, 180$-min insulin level: $249 \mu \mathrm{U} / \mu \mathrm{L})$. Her body mass index was within normal range $\left(24.1 \mathrm{~kg} / \mathrm{m}^{2}\right)$. Fasting glucose was normal until she reached 20 years of age. Thence, due to the persisting extreme insulin resistance, a combined therapy with metformin $(1500 \mathrm{mg} /$ day $)$ and insulin was introduced. At present, she is treated with fastacting insulin (aspart 45IU, three times daily) and longacting insulin (detemir $15 \mathrm{IU}$ at night). In addition, she presented dyslipidemia with severe hypertriglyceridemia $(350 \mathrm{mg} / \mathrm{dL})$, mild hypercholesterolemia $(220 \mathrm{mg} / \mathrm{dL})$ and mild hyperuricemia. At recent examination, BMI remained within normal range, but she showed a mild centripetal fat distribution and a fatty liver at abdominal ultrasonography.

She has a long history of irregular menstrual cycles since adolescence and an estroprogestinic therapy was introduced. At 40 years of age, she developed amenorrhea, which was associated with a hyperprolactinemia (PRL: $999 \mathrm{ng} / \mathrm{mL}$ ) of unknown origin, with a further increasing 6 months later (PRL: $3379 \mathrm{ng} / \mathrm{mL}$ ). Total testosterone, LH, FSH and TSH were in normal range. Brain MRI did not reveal any pituitary lesion (Fig. 1H). At 41 years, she was diagnosed with a bilateral carpal tunnel syndrome and mild sensory motor symmetrical polyneuropathy at lower limbs likely due to diabetes.

\section{Genetic studies}

The proband and her mother provided written informed consent for the molecular analyses, and the study was approved by the Internal Ethics Committee of the Department of Medical Sciences, University of Torino, Italy. Whole-exome sequencing (WES) on the proband was outsourced to BGI-Shenzen using genomic DNA extracted from circulating leukocytes. Targeted enrichment was performed using SureSelect All Exon v4 kit (Agilent), and captured libraries were loaded onto an Illumina HiSeq 2000 platform (Illumina). WES data analysis was performed using an in-house implemented pipeline $(8,9,10)$. High-quality variants were filtered by discarding those with MAF $>0.5 \%$ in ExAC 0.3 and with frequency $>2 \%$ in our in-house database. SnpEff toolbox v4.2 (11) was used to predict the functional impact of variants and retain missense/nonsense/frameshift changes, coding indels and intronic variants at exonintron junctions (within position \pm 8 ). Functional annotation of variants was performed using snpEff, v4.2 and dbNSFP2.9 $(11,12)$.

Based on consanguinity, we assumed an autosomal recessive model of inheritance for the trait, and retained all the homozygous variants located within LoH genomic stretches using Homozygosity Mapper (13) (http://www.homozygositymapper.org), setting 80 as the number of consecutive homozygous SNPs. Variants were then filtered according to their predicted functional impact, retaining those variants with CADD score $>15(12,14)$, and then prioritized taking into account the biological and clinical relevance of individual genes.

Sequence validation and segregation analyses were performed by Sanger sequencing using an ABI 3130XL and the ABI BigDye Terminator Sequencing Kit, V 3.1 (Life Technologies). Sequences were examined using the SeqScape, v2.6 Software (Life Technologies).

\section{Gene expression studies}

Patient's RNA was isolated from PAXgene-stabilized blood sample using the PAXgene blood RNA kit (PreAnalytix). Commercial RNAs extracted from cell lines representing ten different human tissues (Human MTC Panel I, \#636742 and Human Fetal MTC Panel, \#636747, Clontech Laboratories) were used to test tissue-specific expression of the two major POC1A isoforms. cDNA was generated using the MLV Transcriptase (Applied Biosystem) from $1 \mu \mathrm{g}$ of total RNA. To confirm the presence of both $10+$ and 10- POC1A isoforms, patient's cDNA was amplified using primers already described by Chen et al. and PCR product was run on a $2 \%$ agarose gel stained with ethidium bromide. Bands were gelpurified and Sanger sequenced to confirm the presence of exon 9-exon 10 and exon 9-exon 11 junctions. POC1A expression was tested with primers described by Chen et al. Amplified cDNAs were run on a $2 \%$ agarose gel and stained with ethidium bromide. Band intensities from tissues' cDNA were assessed using the ImageLAB software (Bio-Rad). $\beta$-Actin was used as reference to determine POC1A expression levels. The ratio between mRNA isoforms containing (10+) and lacking (10-) exon 10 was also evaluated. 


\section{Results}

\section{Genetic analysis and gene expression studies}

WES statistics are reported in Supplementary Table 1 (see section on supplementary data given at the end of this article). Data annotation predicted 13841 high-quality variants having functional impact (i.e., non-synonymous and splice site changes). Among them, 400 variants passed the public and in-house database filters and were taken into account for further analyses. Variants were then filtered to retain homozygous sequence changes located within LoH regions (Supplementary Table 2) with disruptive impact on protein structure/function predicted with confidence. Prioritization of the retained variants taking into account the biological relevance of individual genes allowed identifying POC1A as the best candidate disease gene. The proband carried a homozygous frameshift variant (c.1047_1048dupC, p.G337Rfs*25, NM_015426) affecting exon 10 of the gene. As expected, genotyping of maternal DNA confirmed the occurrence of the truncating variant at the heterozygous state, according to the recessive pattern of inheritance (Fig. 2A and B).

POC1A gene had previously been reported to cause SOFT syndrome, whose clinical phenotype significantly differs from that of our patient (Table 1). Of note, a single case with a homozygous POC1A mutation and clinical features overlapping those exhibited by the present patient has recently been reported (7) (Table 1). Remarkably, this patient also carried a frameshift mutation in exon 10 of the gene, differing from location of the previously reported POC1A mutations underlying SOFT syndrome (Fig. 2B).

RNA analysis performed on proband's cDNA confirmed the presence of the $10+$ and the 10- POC1A isoforms (Fig. 2C). Expression analysis on adult and embryonic tissues showed that POC1A mRNA level varies considerably among tissues and that the mRNA isoform lacking exon 10 is significantly represented in the POC1A mRNA pool, being from 34\% (adult frontal cortex) to 8.6\% (adult stomach) (Fig. 2C), supporting the hypothesis of a less severe clinical impact of mutations affecting this exon.

\section{Discussion}

Pleiotropy is a common phenomenon in Mendelian diseases, and mutations affecting genes with pleiotropic effects can frequently cause distinct phenotypes. Different molecular mechanisms underlie this phenomenon (15).
The pathogenic variants can affect diverse functional domains of the protein (e.g., PHD-like vs helicase domains in ATRX), have mild or severe impact on protein function (missense vs truncating mutations) or cause quantitatively distinct effects (loss-of-function vs gainof-function mutations). In a few but instructive cases reported in literature, different classes of mutations have been shown to specifically affect distinct isoform of the protein. One example is represented by mutations in PLEC gene (16). Changes affecting exons common to all eight PLEC isoforms cause epidermolysis bullosa simplex (EBS), whereas mutations located in the alternative exon 1a, disrupt specifically the skin-specific isoform of the protein (P1a) and cause a less severe condition in which the disorder is limited to epidermis without extracutaneous involvement. We describe the second patient with a homozygous mutation in the POC1A gene not associated with SOFT syndrome and whose clinical features overlap those of a previous subject reported by Chen et al. Our case confirms that mutations in POC1A gene cause two distinct conditions: SOFT syndrome (6) and a variant phenotype (variant POC1A-related, vPOC1A, syndrome) characterized by extreme insulin resistance and short stature. The main clinical differences between the two 'phenotypes' include an extreme dyslipidemia with insulin resistance and acanthosis nigricans, which represent a major feature in vPOC1A syndrome, but do not occur in SOFT syndrome. Facial dysmorphism is more severe and distinctive of patients with SOFT syndrome, and ectodermal dysplasia has not been observed in the two reported cases with the vPOC1A phenotype (Table 1). SOFT-associated mutations are nonsense $(3,6)$ or missense $(2,5)$ changes that affect all the three POC1A isoforms, causing a complete loss of function (Fig. 2). On the other hand, patients affected by the vPOC1A syndrome carry mutations restricted to exon 10 of the POC1A gene and affect only two of the three POC1A isoforms, maintaining functional the protein lacking exon 10 encoded region (in frame 48 a.a. deletion). The residual function of the protein likely explains the overlapping features of SOFT and vPOC1A syndromes, which are milder in the latter. Furthermore, the insulin-resistant hyperinsulinemia, a distinctive phenotype of vPOC1A patients, may be due to the unbalance of the $10+/ 10-$ isoforms present only in those patients.

Notably, POC1A encodes a protein associated with centrioles during the cell cycle and crucial for both mitotic spindle and primary ciliary function. Other genetic defects affecting the centrosome cause extreme insulin resistance with severe dyslipidemia, 
A

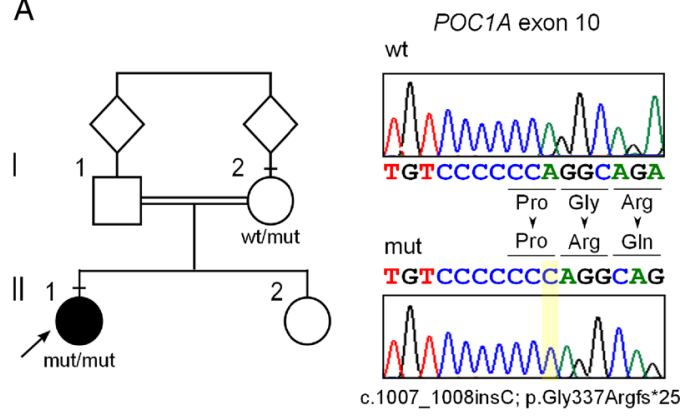

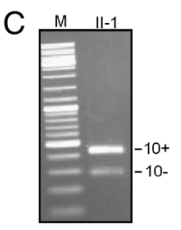
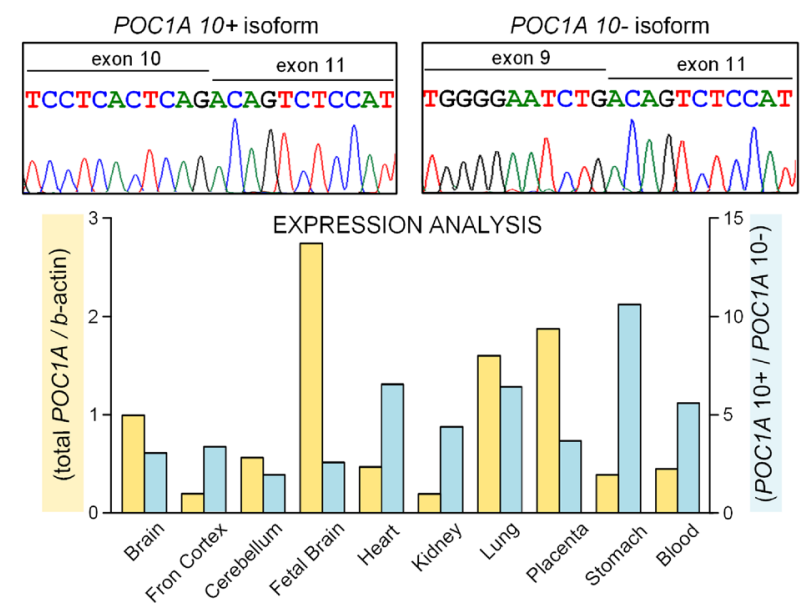

B

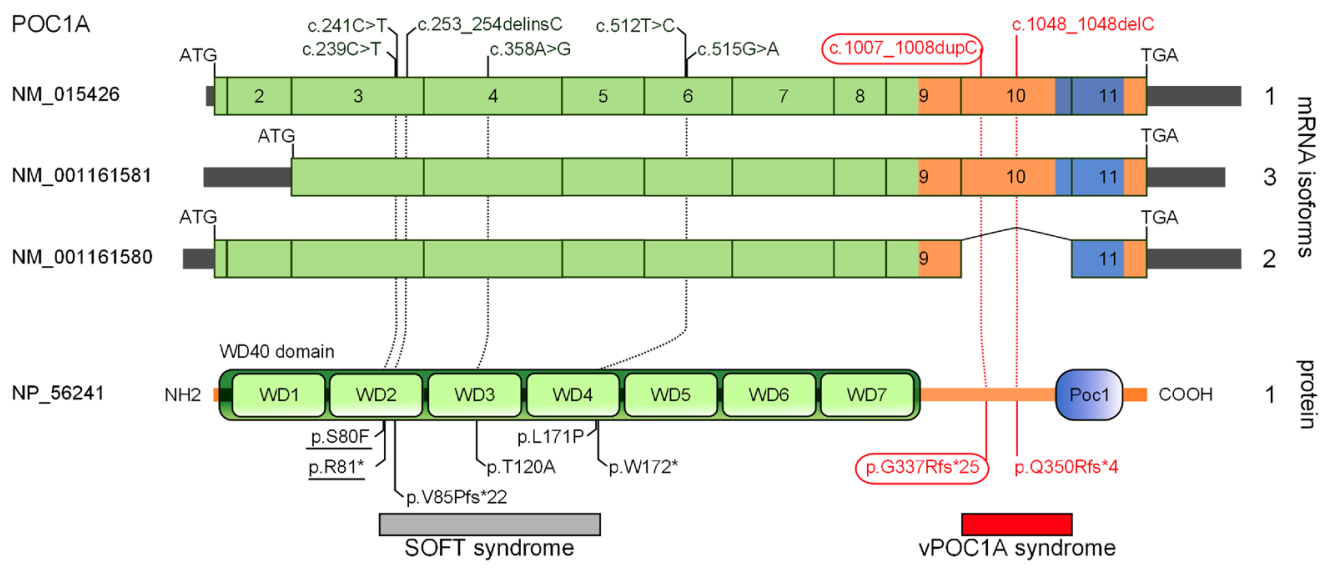

Figure 2

Molecular findings. (A) Pedigree of the family reporting the segregation of the pathogenic variant. Black symbol and arrow indicate the affected case (proband). A line above symbols indicates DNA availability. The sequence chromatogram showing the disease-causing mutation, c.1007_1008dupC (p.Gly337Argfs*25) in the POC1A gene, is reported. (B) Structure of the three cDNA isoforms and predicted protein of $P O C 1 A$ are illustrated. On the left the corresponding accession numbers. Exons of the POC1A gene are distinguished into coding (colored boxes) and non-coding (small black boxes). The corresponding functional domains are shown at protein level ( $\mathrm{N}$-terminal seven-bladed $\beta$-propeller WD40 domain (green); C-terminal coiled-coil domain that includes the highly conserved Poc1 motif (light blue)). All reported pathogenic variants are in homozygous state with the exception of a patient compound heterozygous (underlined). In black, pathogenic changes associated with SOFT syndrome; in red, pathogenic changes associated with IRSS syndrome. The c.1007_1008dupC (p.Gly337Argfs*25) variant identified in our patient is boxed. (C) POC1A CDNA analysis. On the upper part, gel electrophoresis of patient's CDNA showing 10+ and 10- POC1A isoforms. The splicing junctions (exon 9-exon 10 and exon 9-exon 11) were Sanger sequenced. On the lower part, POC1A expression analysis. Histograms indicate tissue-specific expression of total POC1A (in yellow) and the ratio between mRNA isoforms containing (exon 10+) or lacking (exon 10-) exon 10 (in light blue). From left to right, tissue analyzed were human adult brain, adult cerebellum, adult frontal cortex, fetal brain, adult heart, adult kidney, adult lung, adult placenta, adult stomach and adult blood. A full colour version of this figure is available at http://dx.doi.org/10.1530/EJE-17-0431.

such as Alström syndrome (17) and microcephalic osteodysplastic primordial dwarfism type II (MOPDII) (18), supporting a connection between dysregulated function of some centriolar and pericentriolar proteins and defective peripheral response to insulin and adipose tissue maintenance and regeneration.
In conclusion, biallelic mutations in POC1A gene cause two clinically distinct conditions: SOFT syndrome, which is characterized by short stature, onychodysplasia, facial dysmorphism and hypotrichosis, and a less severe phenotype, vPOC1A, major features of which include extreme insulin resistance and short stature and is specifically caused by 


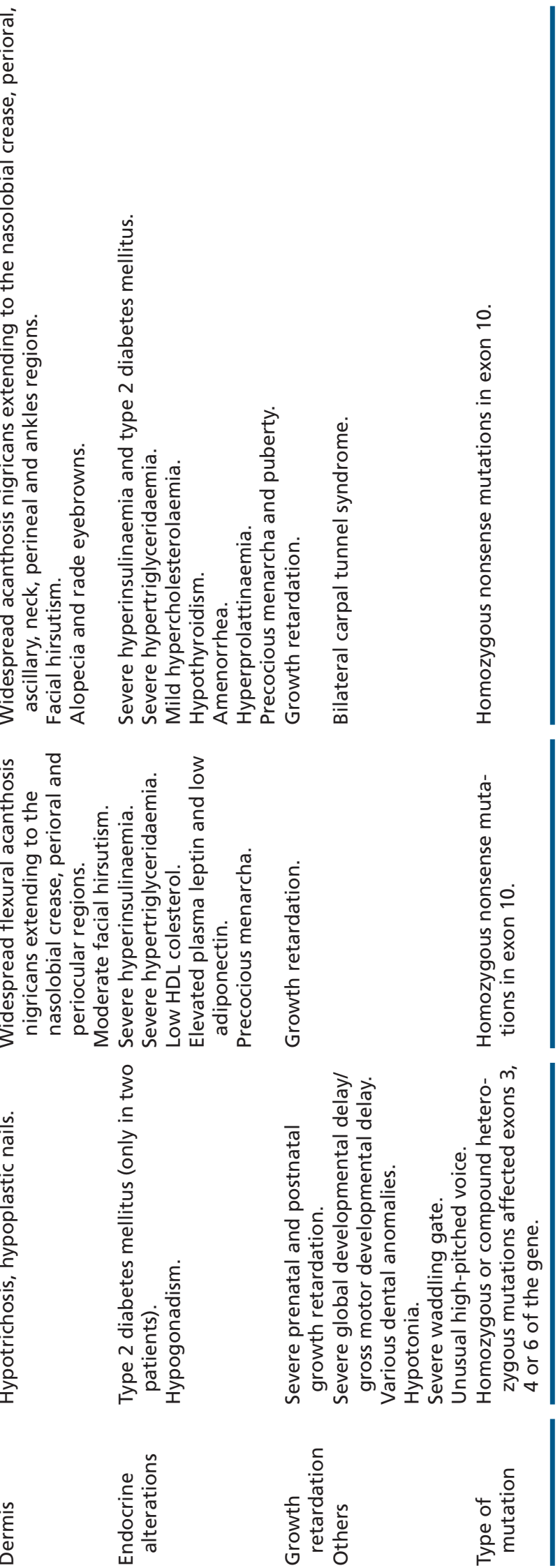


mutations affecting exon 10 of the gene. The present findings point to this specific class of mutations in POC1A as the cause of cases with idiopathic insulin resistance and short stature.

\section{Supplementary data}

This is linked to the online version of the paper at http://dx.doi.org/10.1530/ EJE-17-0431.

\section{Declaration of interest}

The authors declare that there is no conflict of interest that could be perceived as prejudicing the impartiality of the research reported.

\section{Funding}

This work was supported in part by MURST60\%, Associazione 'E.E. Rulfo' (to $A B$ ), and Fondazione Bambino Gesù (to $M T$ ).

\section{Acknowledgment}

\section{References}

1 Venoux M, Tait X, Hames RS, Straatman KR, Woodland HR \& Fry AM. Poc1A and Poc1B act together in human cells to ensure centriole integrity. Journal of Cell Science 2013126 163-175. (doi:10.1242/ jcs.111203)

2 Sarig O, Nahum S, Rapaport D, Ishida-Yamamoto A, Fuchs-Telem D, Qiaoli L, Cohen-Katsenelson K, Spiegel R, Nousbeck J, Israeli S et al. Short stature, onychodysplasia, facial dysmorphism, and hypotrichosis syndrome is caused by a POC1A mutation. American Journal of Human Genetics 201291 337-342. (doi:10.1016/j.ajhg.2012.06.003)

3 Barraza-Garcia J, Ivan Rivera-Pedroza C, Salamanca L, Belinchon A, Lopez-Gonzalez V, Sentchordi-Montane L, del Pozo A, Santos-Simarro F, Campos-Barros A, Lapunzina P et al. Two novel POC1A mutations in the primordial dwarfism, SOFT syndrome: clinical homogeneity but also unreported malformations. American Journal of Medical Genetics Part A 2016 170A 210-216. (doi:10.1002/ajmg.a.37393)

4 Ko JM, Jung S, Seo J, Shin CH, Cheong HI, Choi M, Kim OH \& Cho TJ. SOFT syndrome caused by compound heterozygous mutations of POC1A and its skeletal manifestation. Journal of Human Genetics 2016 61 561-564. (doi:10.1038/jhg.2015.174)

5 Koparir A, Karatas OF, Yuceturk B, Yuksel B, Bayrak AO, Gerdan OF, Sagiroglu MS, Gezdirici A, Kirimtay K, Selcuk E et al. Novel POC1A mutation in primordial dwarfism reveals new insights for centriole biogenesis. Human Molecular Genetics 201524 5378-5387. (doi:10.1093/hmg/ddv261)

6 Shaheen R, Faqeih E, Shamseldin HE, Noche RR, Sunker A, Alshammari MJ, Al-Sheddi T, Adly N, Al-Dosari MS, Megason SG et al. POC1A truncation mutation causes a ciliopathy in humans characterized by primordial dwarfism. American Journal of Human Genetics 201291 330-336. (doi:10.1016/j.ajhg.2012.05.025)

7 Chen JH, Segni M, Payne F, Huang-Doran I, Sleigh A, Adams C, Consortium UK, Savage DB, O'Rahilly S, Semple RK et al. Truncation of POC1A associated with short stature and extreme insulin resistance. Journal of Molecular Endocrinology 201555 147-158. (doi:10.1530/JME-15-0090)

8 Cordeddu V, Redeker B, Stellacci E, Jongejan A, Fragale A, Bradley TE, Anselmi M, Ciolfi A, Cecchetti S, Muto V et al. Mutations in ZBTB20 cause Primrose syndrome. Nature Genetics 201446 815-817. (doi:10.1038/ng.3035)

9 Kortum F, Caputo V, Bauer CK, Stella L, Ciolfi A, Alawi M, Bocchinfuso G, Flex E, Paolacci S, Dentici ML et al. Mutations in KCNH1 and ATP6V1B2 cause Zimmermann-Laband syndrome. Nature Genetics 201547 661-667. (doi:10.1038/ng.3282)

10 Niceta M, Stellacci E, Gripp KW, Zampino G, Kousi M, Anselmi M, Traversa A, Ciolfi A, Stabley D, Bruselles A et al. Mutations impairing GSK3-mediated MAF phosphorylation cause cataract, deafness, intellectual disability, seizures, and a down syndrome-like facies. American Journal of Human Genetics 201596 816-825. (doi:10.1016/j. ajhg.2015.03.001)

11 Cingolani P, Platts A, Wang le L, Coon M, Nguyen T, Wang L, Land SJ, Lu X \& Ruden DM. A program for annotating and predicting the effects of single nucleotide polymorphisms, SnpEff: SNPs in the genome of Drosophila melanogaster strain w1118; iso-2; iso-3. Fly 20126 80-92. (doi:10.4161/fly.19695)

12 Liu X, Jian X \& Boerwinkle E. dbNSFP v2.0: a database of human nonsynonymous SNVs and their functional predictions and annotations. Human Mutation 201334 E2393-E2402. (doi:10.1002/humu.22376)

13 Seelow D \& Schuelke M. HomozygosityMapper2012 - bridging the gap between homozygosity mapping and deep sequencing. Nucleic Acids Research 201240 W516-W520. (doi:10.1093/nar/gks487)

14 Kircher M, Witten DM, Jain P, O'Roak BJ, Cooper GM \& Shendure J. A general framework for estimating the relative pathogenicity of human genetic variants. Nature Genetics 201446 310-315. (doi:10.1038/ng.2892)

15 Zhu X, Need AC, Petrovski S \& Goldstein DB. One gene, many neuropsychiatric disorders: lessons from Mendelian diseases. Nature Neuroscience 201417 773-781. (doi:10.1038/nn.3713)

16 Gostynska KB, Nijenhuis M, Lemmink H, Pas HH, Pasmooij AM, Lang KK, Castanon MJ, Wiche G \& Jonkman MF. Mutation in exon 1a of PLEC, leading to disruption of plectin isoform 1a, causes autosomalrecessive skin-only epidermolysis bullosa simplex. Human Molecular Genetics 201524 3155-3162. (doi:10.1093/hmg/ddv066)

17 Collin GB, Marshall JD, Ikeda A, So WV, Russell-Eggitt I, Maffei P, Beck S, Boerkoel CF, Sicolo N, Martin M et al. Mutations in ALMS1 cause obesity, type 2 diabetes and neurosensory degeneration in Alstrom syndrome. Nature Genetics 200231 74-78. (doi: 10.1038/ng867)

18 Willems M, Genevieve D, Borck G, Baumann C, Baujat G, Bieth E, Edery P, Farra C, Gerard M, Heron D et al. Molecular analysis of pericentrin gene (PCNT) in a series of $24 \mathrm{Seckel} / \mathrm{microcephalic}$ osteodysplastic primordial dwarfism type II (MOPD II) families. Journal of Medical Genetics 201047 797-802. (doi:10.1136/jmg.2009.067298)

Received 25 May 2017

Revised version received 27 July 2017

Accepted 17 August 2017 